\title{
Fractal and Voltammetric Study of Linoleic Acid Adsorption at the Mercury/Electrolyte Solution Interface
}

\author{
D. Risović*, B. Gašparovićt and B. Ćosović ${ }^{+}$ \\ ${ }^{*}$ Molecular Physics Laboratory and ${ }^{+}$Center for Marine and Environmental Research, \\ Ruđer Bošković Institute, POB 180, HR-10002 Zagreb, Croatia
}

\begin{abstract}
A new method, size-scaling of the hanging mercury drop electrode, is introduced and used to determine the fractal properties of the adsorbed layer and its dependence on fractional electrode coverage. Simultaneously, the adsorption process of linoleic acid at a mercury electrode surface has been studied by means of phase-sensitive ac voltammetry in $0.5 \mathrm{M} \mathrm{NaCl}$ electrolyte solution, $\mathrm{pH} 8.3$, simulating seawater conditions. Three concentration domains that exhibit different fractal behavior characterize the adsorption process. In the first domain, corresponding to the fractional electrode coverage $\theta<0.5$, the fractal dimension of the adsorbed layer $\mathrm{D} \leq 2$. Further increase in surface concentration (electrode coverage) is followed by a swift change in fractal dimension, reaching the peak value $\mathrm{D} \approx 2.4$. This indicates that, at fractional electrode coverage of $0.7-0.8$, depending on adsorption conditions, a second order phase change in the adsorbed layer of linoleic acid takes place. In the third domain, the fractal dimension rapidly decreases to $\mathrm{D} \approx 2.0$ and remains stable regardless of bulk concentration increase, reflecting formation of a rather uniform and homogenous layer of perpendicularly oriented molecules of linoleic acid. The observed changes of fractal dimension are result of restructuring of the adsorbed layer and
\end{abstract}


a change in the orientation of adsorbed molecules due to molecular interactions. The influence of electrode polarity on the adsorption process of linoleic acid and fractal properties of the adsorbed layer is discussed. Comparison with the apparent isotherm, obtained in a classical voltammetric way, shows that the analysis of fractal properties of the adsorbed layer provides a useful tool for studies of the adsorption process and a sensitive method for detection of phase changes.

\section{Introduction}

Lipids, as well as fatty acids, play an important role in natural aquatic systems. Due to their amphiphatic molecular structure, they adsorb on natural phase boundaries, influencing mass and energy exchange. Hydrophobic chain plays a role in hydrophobic interactions and leads to low aqueous solubility, while the functional group makes lipids suitable for electrostatic interactions. They easily form films on the air/water interfaces and may considerably influence the gas exchange on such a modified interface. ${ }^{1}$ Due to the large sorption capacity and amphiphatic molecular structure of fatty acids, they are found in sea water mostly in the particulate form, where concentrations are up to $5-10$ times higher than in the dissolved phase. ${ }^{2}$

The adsorption of organic molecules and its various aspects have been extensively studied for a long time. ${ }^{3-8}$ Lipids are frequently used to study organized monolayers on air/water or mercury electrode/water interfaces ${ }^{9-11}$, since air and mercury electrode are both hydrophobic phase boundaries having similar adsorption effects on many organic substances. $^{12}$ 
Adsorption at a surface is conveniently studied by electroanalytical methods. One of such frequently used methods is the phase sensitive ac voltammetry. Organic molecules adsorbing on the electrode cause changes of the electric double layer and hence a decrease in capacitive current. ${ }^{13,14}$ A direct measure of the amount of an organic substance adsorbed at the electrode is given by the total capacitance of the electrode/solution interface. Since the measurable capacitive current is directly proportional to the capacitance, the adsorption process can be monitored directly and continuously. The measured data can be used to construct the adsorption isotherm and to determine its parameters. However, sometimes this is not a straightforward and simple procedure due to the structure of adsorbates and the complexity of involved interactions.

Adsorption of fatty acids on mercury electrode is dominated by their hydrophobic properties. Adsorption experiment with fatty acids with an increasing chain length showed good correlation between the adsorption constant B and the octanol/water coefficient, as a measure of the hydrophobic properties. ${ }^{15}$ Generally, adsorption on the electrode is dependent on the concentration, but also on the applied potential. Adsorption experiment with sodium oleate, ${ }^{16}$ using three dimensional phase sensitive ac voltammetry, showed that at moderate and negative polarization of mercury electrode for the bulk concentrations below critical micellar concentration $\left(\mathrm{cmc}=2 \times 10^{-4} \mathrm{M}\right.$ at $\left.25^{\circ} \mathrm{C}\right),{ }^{16}$ sodium oleate formed a close-packed monolayer. This film consisted of close-packed patches rather than a compact layer. At postmicellar bulk concentrations, multilayer adsorption occurred throughout the potential range studied. ${ }^{16}$ Furthermore, models of lipid phase transitions ${ }^{6,7}$ predict a rather complicated reorientation, whereby the lipid monolayer reverts first to a two-phase system of a thick and a thin monolayer, and finally to a pored bilayer. Sometimes, for relatively large negative potentials, the adsorbed layers can be in fact displaced from the mercury surface to form aggregate structures near the electrode surface, which respread at less 
negative potentials. ${ }^{11}$ All this indicates that, in addition to the commonly used methods, some other tools should be also used to deal with such complexities.

Fractal geometry proved to be a useful tool in dealing with physical and chemical problems that involve non-trivial geometries. Such geometries are in fact more common in the physical world than the classical Euclidean geometries, especially if we consider surfaces and porous structures such as aggregates or fractured objects. Some of the chemical topics studied by fractal analysis include: surface accessibility to physisorption of small molecules, ${ }^{17,18}$ surface-geometry effects on the adsorption of polymers, ${ }^{19,20}$ estimation of the surface available for reactions between adsorbates, ${ }^{21,22}$ particle size effects in physisorption and chemisorption, ${ }^{23}$ surface geometry effects on reactions with catalytic and non-catalytic fractal surfaces, ${ }^{24-26}$ growth of layers on mercury electrode. ${ }^{27}$

The fractal analysis approach is based on the possibility to describe quantitatively complex objects that are statistically scale-invariant, physical realizations of mathematical fractals that appear the same on all length scales. This property is manifested in a powerlaw dependence of the density-density correlation function $g(r) \sim r^{D-d}$, where $d$ and $D$ denote spatial and fractal dimensions, respectively.

More generally, a power-law-scaling ratio characterizes one or more of the features of an object or a process carried out near or at the object:

$$
\text { Feature } \sim \text { scale }^{\Delta}
$$

Here "feature" should be considered in the broadest sense: it could be the surface area, scattered light intensity, the rate of heterogeneous reaction or the shape of adsorption isotherm. The "scale" can be particle size, pore diameter, scattering vector, cross-sectional area of an adsorbate or layer thickness. The exponent $\Delta$ is a parameter, which indicates how sensitive the considered feature is to changes in the applied scale. 
This non-integer exponent $\Delta$, originally inferred from relations between purely geometric parameters and "yardsticks" used to determine them, has the meaning of dimension, which Mandelbrot ${ }^{28}$ termed "fractal". Extension of this concept, coming from the recognition that effective geometries of various structures and processes can be described in terms of fractal geometry, results in an effective fractal dimension $\mathrm{D}_{\text {feature }}$ or simply D.

However, in adsorption studies, fractal analysis was so far applied mostly to the surface and it dealt with the fractality of the surface and its influence on the adsorption process and not with the fractal structure of the adsorbed layers itself. Hence, our aim was to develop a method for determining fractality of the adsorbed layer and then to apply fractal analysis to the adsorption process in order to gain more insight and information than are readily obtained by classical methods.

Here, we present a new method for estimation of the fractal dimension of the adsorbed layer and the results of simultaneous ac voltammetric and fractal analysis of the adsorption process of linoleic acid (LA) at a hanging mercury drop electrode from $0.55 \mathrm{M}$ solution of $\mathrm{Na} \mathrm{Cl}$, and for the concentration range of LA of $0-70 \mathrm{mg} / \mathrm{dm}^{3}\left(0-2.5 \times 10^{-4} \mathrm{~mol} / \mathrm{dm}^{3}\right)$.

\section{Theory}

The extent of adsorption of organic substances on electrode surface depends on their concentration, surface potential and molecular interactions. The nonpolar molecules adsorb more strongly on the mercury electrode when the electrode surface is near zero potential charge while at substantial positive or negative surface charges, the nonpolar molecules at the surface are replaced by polar ones. This is reflected in a change of capacitive current 
values of the electrode/solution interface as a function of the applied potential. The simplest model of capacitive behavior of the electrode/solution interface is that of serially coupled capacitors ${ }^{29}\left(\mathrm{C}_{1}\right.$ and $\left.\mathrm{C}_{2}\right)$, representing spaces bounded by inner and outer Helmholtz planes. In such system, the layer with the lowest capacitance practically governs the total capacitance.

If organic molecules become adsorbed, this analogue circuit model is modified in such a way that a third capacitor, $\mathrm{C}_{3}$, is added in parallel to the interface capacity. $\mathrm{C}_{3}$ represents the specific capacity of the surface covered by organic molecules. Consequently, taking into account that $\mathrm{C}_{1}<<\mathrm{C}_{2}$, the capacitance is given by

$$
C \cong(1-\theta) C_{1}+\theta C_{3}
$$

here $\theta$ represents the fractional electrode surface coverage with organic adsorbates.

Since the capacitive current is proportional to the differential interface capacitance, this simple model permits calculation of the surface fractional coverage $\theta$ directly from the measured capacitive current: ${ }^{12}$

$$
\theta=\frac{i_{0}-i_{c}(\theta)}{i_{0}-i_{c}(\theta=1)}
$$

here io represents the capacitive current corresponding to the interface capacitance without organic molecules, $\mathrm{i}_{\mathrm{c}}(\theta)$ the capacitive current in the presence of adsorbed organic molecules and $\mathrm{i}_{\mathrm{c}}(\theta=1)$ the capacitive current obtained for the totally covered electrode.

To describe the adsorption process and to account for the relationship between the amount of adsorbed species on electrode surface, surface coverage and its corresponding activity in bulk solution, a variety of adsorption isotherms have been proposed. ${ }^{12}$ The main difference between them is the type of the included adsorbate-adsorbate interaction. A frequently used one is the Frumkin isotherm given by: 


$$
\frac{\theta}{1-\theta} e^{-2 a \theta}=B c
$$

Here, $a$ is the interaction coefficient, $c$ is the equilibrium bulk concentration of the adsorbate and $\mathrm{B}$ is the adsorption constant with dimension $\mathrm{cm}^{3} / \mathrm{mol}$.

The interaction coefficient influences the shape of the adsorption isotherm and reflects the intensity of lateral interactions of the adsorbate at the surface. If $a>0$, lateral interactions are attractive, while interactions are repulsive for $\mathrm{a}<0$. A tacit assumption made in Eq. (4) is that, to a good approximation, appropriate bulk concentrations can be used in place of adsorbate activity.

One way of obtaining the interaction coefficient in the Frumkin-type isotherm is to follow the non-thermodynamic procedure outlined by Damaskin. ${ }^{12}$ First, the concentration of the organic substance corresponding to $\theta=0.5$ is found, and the experimental isotherm is re-plotted as $\theta$ vs. $\mathrm{c} / \mathrm{c}_{\theta=0.5}$. Then, using the relative concentration and dividing Eq. (4) by $\mathrm{Bc}_{\theta=0.5}=\mathrm{e}^{-\mathrm{a}}$, the equilibrium adsorption constant $\mathrm{B}$ can be eliminated from the equations, yielding:

$$
y=\frac{c}{c_{\theta=0.5}}=\frac{\theta}{1-\theta} e^{a(1-2 \theta)}
$$

Solving for $a$, we find:

$$
a=\frac{2.3}{1-2 \theta} \log \left[\frac{y(1-\theta)}{\theta}\right]
$$

Substitution of various values of $\theta$ and y from the experimental $\theta$ vs. y curve gives the corresponding values of the interaction coefficient. 
To obtain the fractal dimension of the system, we rely on relation (1) where the "feature" used is the capacitive current at a selected potential, and the scale is the size (radius $r$ ) of the hanging mercury drop electrode. The total differential capacity of interface $\mathrm{C}_{\mathrm{t}}$, inferred from (2), is given by

$$
C_{t}=(1-\theta) A C_{1}+\theta A C_{3}
$$

where $\mathrm{A}$ is the electrode surface.

Since $\mathrm{I}_{\mathrm{c}} \sim \mathrm{C}_{\mathrm{t}}$, and $\mathrm{A} \sim r^{\mathrm{D}}$, we obtain

$$
\mathrm{Ic} \sim r^{\mathrm{D}}
$$

Hence, the fractal dimension $\mathrm{D}$ can be obtained from the slope of the log-log plot of $\mathrm{I}_{\mathrm{c}} \mathrm{vs}$. $r$.

If the electrode surface is smooth, the current should scale linearly with the area, i.e. $\mathrm{I}_{\mathrm{c}}$ $\sim r^{2}$. In this case the fractal dimension equals the classical Euclidean dimension. However if the morfology of the surface is changed in a some way from "smooth and uniform" to "irregular", but self-similar, this will be reflected in a different fractal dimension Hence, since a small mercury drop represents an ideally smooth and spherical surface, the presence of the adsorbed molecular layer will be reflected in the change of fractal dimension. In the case when the whole electrode surface is covered with an ideal homogenous adsorbed layer relation (8) should give $\mathrm{D}=2.0$. However, if the surface is covered either partialy or totaly with an layer that has not an uniform but fractal structure the fractal dimension shall reflect this self-similar nonuniformities and be different from $\mathrm{D}=2$.

\section{Experimental section}

For the study of adsorption of LA, phase sensitive ac voltammetry ( $90^{\circ}$ out of phase) was used. Ac voltammetric measurements were performed by AUTOLAB with PGSTAT 20 (Ecochemie, Netherlands). The frequency of the ac voltage was $170 \mathrm{~Hz}$, the amplitude 
$0.010 \mathrm{Vrms}$, step potential $0.0021 \mathrm{~V}$, interval time $0.29 \mathrm{~s}$ and modulation time $0.19 \mathrm{~s}$. All experiments were performed in a three-electrode system with a hanging mercury drop electrode produced by Metrohm (Switzerland). $\mathrm{Ag} / \mathrm{AgCl}$ electrode was used as the reference electrode and a platinum wire as the auxiliary electrode. In order to increase the measurement sensitivity, LA was accumulated under stirring of the solution (270 r.p.m.), at a potential of $-0.35 \mathrm{~V}, 1$ min prior to potential scan. Since the structure of the adsorbed layer is strongly influenced by the electrode potential and surface charge, the electrode potential in the experiment was varied in the relevant range of $\mathrm{E}=-0.35 \mathrm{~V}$ to $-0.90 \mathrm{~V}$, with resolution $\approx 2 \mathrm{mV}$. The lower limit of the range is imposed by the fact that, at the more negative electrode potentials desorption of LA occurs. Also, this potential range selection eliminates the pseudocapacitive currents. The results were elaborated for three electrode potentials, $-0.6 \mathrm{~V},-0.8 \mathrm{~V}$ and $-0.35 \mathrm{~V}$, representing non-polar and polarized electrodes.

For the purpose of the voltammetric analyses the measurements were performed in the potential range $\mathrm{E}=-0.35 \mathrm{~V}$ to $-1.75 \mathrm{~V}$ The values of differential capacitance were calculated from the measured capacity current. The calibration was done using a series of known capacitors (precision $\pm 1 \%$ ).

All measurements were repeated three times in freshly prepared solutions. The adsorption of LA was studied for a concentration range of $0-70 \mathrm{mg} / \mathrm{dm}^{3}\left(0-2.510^{-4} \mathrm{M}\right)$.

For the purpose of fractal analyses in the voltammetric measurements the volume of the mercury drop electrode was varied stepwise over nine sizes in the range of 0.08 to 0.7 $\mathrm{mm}^{3}$, which corresponds to the electrode surface area in the range of 0.9 to $3.8 \mathrm{~mm}^{2}$.

For every investigated concentration of linoleic acid the voltammetric measurements were performed with nine different drop sizes, each times with a freshly prepared new drop of a selected size. During one voltammetric measurement the drop size was not changed. 
Linoleic acid (Fluka, Switzerland) was used without further purification. Mercury was purified by double distillation under reduced pressure. $\mathrm{NaHCO}_{3}$ (Merck) was used without prior purification. $\mathrm{NaCl}$ (Kemika, Croatia) was purified by prolonged heating at $450^{\circ} \mathrm{C}$. All solutions were prepared with deionised water obtained with the Milly-Q Water System (Millipore, Switzerland). Carbonate buffer was used to maintain $\mathrm{pH}$ 8.3. All solutions simulated seawater conditions $\left(0.5 \mathrm{M} \mathrm{NaCl}, 2 \times 10^{-3} \mathrm{M} \mathrm{NaHCO}_{3}\right.$, and $\left.\mathrm{pH} 8.3\right)$.

\section{Results and discussion}

The capacitive current data obtained by phase sensitive ac voltammetry were subjected to a fractal analysis in order to determine the fractal dimension of the adsorbed layer, depending on the electrode potential and concentration of the LA solution. Simultaneously the apparent isotherms were constructed and analyzed.

The change of differential capacity with applied potential is shown in Fig.1. For the lower range of investigated concentrations of LA $\left(<3 \mathrm{mg} / \mathrm{dm}^{-3}\right)$ two desorption peaks were recorded at $\mathrm{E} \approx-1.1 \mathrm{~V}$ and $-1.35 \mathrm{~V}$, respectively. For the concentrations above $3 \mathrm{mg} / \mathrm{dm}^{-3}$ LA only one desorption peak was recorded at $\mathrm{E} \approx-1.35 \mathrm{~V}$.

Here we present results elaborated for the three selected electrode potentials, namely, $0.6 \mathrm{~V},-0.35 \mathrm{~V}$ and $-0.8 \mathrm{~V}$, representing approximately non-polar and polarized electrodes.

The variation of differential capacity with LA concentration for the selected potentials is shown in Fig.2. Capacitance minimum values are 2.4, 2.5 and $3.11 \mu \mathrm{F} / \mathrm{cm}^{2}$, respectively.

The typical capacitive currents vs. potential curves for nine electrode drop sizes are shown in Fig 3. The fractal dimension was determined from the slope of the log-log plot of $i_{c}$ vs. $r$. 
The average fractal dimension determined from measurements in pure electrolyte is D $=1.99 \pm 0.02$, reflecting the absence of the adsorbed molecular layer on a smooth electrode surface. The excellent linear fit (regression coefficient 0.9998 ) of measured data in log-log plot of $i_{c}$ vs. $r$ (Fig. 4, data set 1), exhibiting $\mathrm{r}^{2}$ dependance, for pure electrolyte indicates that the drop remains spherical for all used electrode sizes. The linearity of fit is preserved to high degree in all measurements independently on LA concentration (cf. Fig. 4, data set 2), indicating that change of Hg-drop surface tension due to adsorbed LA does not affect the sphericity of the drop.

First, we consider the adsorption process of LA at the electrode potential of $-0.6 \mathrm{~V}$. This potential approximately corresponds to a non-polar electrode, for which the adsorption of organic matter is most efficient. ${ }^{12}$ The change of the fractal dimension with increasing the LA concentration is shown in Fig. 5a. It can be seen that the presence of even a very low concentration of LA in the electrolyte, and hence the occurrence of adsorbate on the electrode surface, is reflected in an immediate change of the fractal dimension.

Considering the change in the fractal dimension with concentration increase, one can readily identify three different domains related to concentration range and corresponding behaviour of fractal dimension. In the first domain, i.e. in a very low concentration range $\left(C<0.1 \mathrm{mg} / \mathrm{dm}^{3}\right)$, the fractal dimension slowly declines from the initial $\mathrm{D}=2$ for clean electrode to a minimum of $\mathrm{D} \approx 1.89$ and then, with further increase of surface coverage, it gradually increases to $\mathrm{D} \approx 2$.

The second domain, lying roughly between $\mathrm{C}=0.1-1 \mathrm{mg} / \mathrm{dm}^{3}$, is characterized by abrupt and pronounced changes in fractal dimension. With an increase of concentration, the fractal dimension jumps to a peak value of $\mathrm{D} \approx 2.4$, followed by a quick decrease and slight oscillation around $\mathrm{D} \approx 2$. 
In the third domain $\left(\mathrm{C}>1 \mathrm{mg} / \mathrm{dm}^{3}\right)$, the fractal dimension is stable at $\mathrm{D} \approx 2$ $(1.97 \pm 0.02)$ and practically independent of concentration, reflecting a rather uniform adsorbate layer covering the whole electrode surface. It seems that in this concentration range the rate and conditions of adsorption do not allow much freedom in formation of adsorbed layer but favor a dense uniform packing of molecules.

The behavior of the fractal dimension for the LA concentration in the range $\mathrm{C}<0.1$ $\mathrm{mg} / \mathrm{dm}^{3}$ indicates coverage of the electrode surface, first with separated molecules and "patches" and then the build-up of a more uniform, although not totally compact, molecular monolayer. The abrupt jump of the fractal dimension to $\mathrm{D}=2.4$, for the LA concentration of $\mathrm{C} \approx 0.2 \mathrm{mg} / \mathrm{dm}^{3}$, corresponding to $\sim 70-80 \%$ of electrode coverage (Fig. $5 b)$, indicates that a phase change occurs at this concentration and experimental conditions.

We interpreted this as lifting of flat-lying molecules from the surface and clustering, resulting in a highly disordered layer with a high fractal dimension. At higher concentrations this layer undergoes fast restructuring due to molecular reorientation, reflected in a swift decrease of the fractal dimension. Hence, we concluded that this change in fractal dimension is the consequence of molecular reorientation, what may be classified as a second-order phase change. ${ }^{30,31}$ When the reorientation of molecules is finished, the electrode surface is completely covered with a relatively smooth and stable molecular layer with $\mathrm{D} \approx 2$, in which the molecules are adsorbed perpendicularly respect to the electrode surface. Similar effect, a vertical orientation of adsorbed aliphatic alcohols, even at incompletely covered mercury electrode surface, was observed earlier. ${ }^{5}$ Further increase of concentration does not change this situation, as indicated by the constant value of fractal dimension.

The corresponding fractional electrode coverage $\theta$ is shown in Fig. 5b. It was determined from relation (3) from the measured capacitive current $i_{c}$ vs. LA concentration. 
It is worthwhile pointing out that an examination of this apparent isotherm does not reveal any pronounced change that would indicate that a second-order phase change takes place. An exception is a slight "knee" in the narrow concentration range at $\mathrm{C} \approx 0.1$, which, especially if the measurement points are not closely spaced, might be misinterpreted as an experimental data scatter. However, the change in fractal dimension is much more pronounced and spread over a much broader concentration range, thus providing a more sensitive means for phase-change detection.

To represent a system in which, due to a molecular interaction and a $\pi$-electron interaction with the electrode surface, the organic substance might be adsorbed at the electrode in two different positions (parallel or perpendicular to the surface), as indicated by changes in $\mathrm{D}$, the two-capacitor model must be replaced by a model consisting of three parallel capacitors ${ }^{12}$. In this model, water molecules are placed between the plates of the first, organic molecules with one orientation between the plates of the second, and molecules in the second orientation between the plates of the third capacitor.

If we denote the coverage by organic molecules adsorbed in position 1 (flat) and 2 (perpendicular) by $\theta_{1}$ and $\theta_{2}$, respectively, then it can be shown (see Appendix) that:

$$
\theta_{1}=\theta(1-\theta) \text { and } \theta_{2}=\theta^{2}
$$

where $\theta=\theta_{1}+\theta_{2}$ is the fractional electrode surface coverage.

The fractional electrode coverage with molecules adsorbed in flat and perpendicular orientation, calculated from (9) on the basis of the experimentally determined surface coverage $\theta$, is also shown in Fig. $5 b$ (curves $\theta_{1}$ and $\theta_{2}$ ). From this plot, it is readily seen that the fractal dimension increase coincides with the transition point corresponding to the 
fractional electrode coverage of $\theta \approx 0.5$, from which point onward the perpendicular orientation of adsorbed molecules prevails. The sharp decline of the fractal dimension after the peak value at $\theta \approx 0.85$ coincides with the sharp decrease in the number of flat adsorbed molecules, representing a fast transition into a relatively uniform layer of perpendicularly oriented molecules for fractional coverage $\theta>0.9$.

Dependence of the coefficient of lateral interaction on concentration was calculated with the aid of Eq. (6) and is shown in Fig 5c. Due to the non-equilibrium conditions in the experiment, the results, although obtained using a nonthermodynamic method, should be interpreted cautiously. However, we think that they do reflect, at least qualitatively, the behavior of lateral interaction between the molecules during the adsorption process.

At the onset of adsorption, at a low fractional electrode coverage, the attractive interaction between adsorbate molecules decreases. Further adsorption leads to an abrupt change in the coefficient of interaction, which exhibits fast changes accompanying the restructuring of the adsorbed layer. At a still higher electrode coverage, as the molecules in the adsorbed layer stabilize in perpendicular position, the coefficient of interaction tends to stabilize at a value of $a \approx 1.2$, reflecting completion of the second order phase change, and formation of a relatively homogenous adsorbed layer with $\mathrm{D} \approx 2$. In this perpendicular position, only methyl groups weakly bind the molecules to the electrode surface and the dominant interaction is the hydrophobic attraction between LA chains.

Under the experimental conditions $(\mathrm{pH} 8.3)$, the LA molecule is polar $(\mathrm{pK}=7.08)^{32}$ and we may expect that the relative polarity of the electrode should also play a certain role in the development and structuring of the adsorbed layer. To investigate this, we have also analyzed the measured capacitive current for the electrode potentials $-0.35 \mathrm{~V}$ and $-0.8 \mathrm{~V}$, representing positively and negatively charged mercury electrode surfaces, respectively. Variation of the fractal dimension with the increase in the bulk concentration of LA 
obtained for different electrode potentials is shown in Fig. 6a. It can be seen that, for all investigated electrode potentials, the change of fractal dimension shows a similar trend, but the magnitude of maximal change in $\mathrm{D}$ increases with the change from the negatively to a more positively charged electrode surface. These changes in fractal dimension are accompanied by the corresponding changes in the coefficient of lateral interaction shown in Fig. 6c. The change of the total fractional electrode coverage with LA concentration for the considered electrode potentials is shown in Fig 6b. The change of fractal dimension with respect to the fractional electrode coverage for polarized and neutral electrode indicates that polarization of the electrode shifts the position of the fractal dimension peak toward lower surface coverage. Hence, in contrast to the adsorption process at a neutral electrode, the additional electrostatic interactions at polarized electrode causes the phase change to occur earlier, i.e. at lower fractional electrode coverage.

Oscillations in the magnitude of $\mathrm{D}$ during adsorption indicate reorientation of molecules and restructuring of the adsorbed layer due to a variable interaction between LA molecules and interaction with the polarized electrode. These interactions involve a highly hydrophobic attraction between LA chains and the hydrophobic mercury electrode, but also an electrostatic interaction between the "heads" themselves and the "heads" and electrode surface in the case of the polarized electrode. Hence, the coefficient of lateral interaction also changes with electrode polarity. This dependence is shown in Fig. 6c. At the lowest surface coverage, the coefficient of lateral interaction is attractive and $\approx 1$, and then it decreases to negative values reaching the minimum at $\mathrm{C} \approx 0.1 \mathrm{mg} / \mathrm{dm}^{3}$ for the polarized electrode and at $\mathrm{C}=0.17 \mathrm{mg} / \mathrm{dm}^{3}$ for the neutral electrode. This minimum is rather narrow and it corresponds to the first abrupt increase in D. It is most pronounced for the negatively polarized electrode $(-0.8 \mathrm{~V})$, and less pronounced for $\mathrm{E}=-0.6$ and $-0.35 \mathrm{~V}$. This is in agreement with the prediction of a change of $a$ with potential due to variation of 
the area per adsorbed molecule with the increase of the amount of adsorbed material. ${ }^{12}$ For the concentration range $\mathrm{C}=0.1-0.2 \mathrm{mg} / \mathrm{dm}^{3}$ where the fractal dimension jumps to its peak value, the coefficient of lateral interaction also swiftly increases to a positive value $>1$ and then stabilizes at values $\approx 1.5-2$, corresponding to a more or less uniform layer of perpendicularly adsorbed molecules. These values of coefficient $a$ reflect a stronger hydrophobic attraction between the "tails" of perpendicularly oriented LA molecules in the layer. The interaction coefficient value is higher for the positively polarized electrode than for negatively polarized or neutral electrodes.

For polarized electrode surfaces, the fractional coverage plot shows that equal coverage with flat and perpendicularly oriented molecules occurs at a higher concentration compared to the neutral electrode (Fig 7). For positively and negatively charged electrode surfaces an equal fractional coverage of flat and perpendicularly adsorbed molecules $\left(\theta_{1}=\theta_{2}\right)$ is achieved at $C=0.17 \mathrm{mg} / \mathrm{dm}^{3}$ and $C=0.15 \mathrm{mg} / \mathrm{dm}^{3}$ compared to $C=0.1$ $\mathrm{mg} / \mathrm{dm}^{3}$, in the case of the neutral electrode (Fig. 7a). This indicates that, for polarized electrodes, additional electrostatic interactions between molecular "heads" and electrode surface charge are in favor of flat adsorption of LA at an early stage of the adsorption process. 


\section{Conclusions}

Ac voltammetry and fractal analysis, based on the new method the size-scaling of hanging mercury drop electrode, were used to study an adsorption process of linoleic acid at the mercury /electrolyte solution interface. The fractal dimension of the adsorbed layer was determined for different electrode polarity and in dependence on the LA concentration in solution.

Fractal analysis revealed that, depending on electrode polarity, a phase change of the second order occurs at the fractional electrode coverage $\theta=0.7-0.8$. This phase change is a consequence of interactions resulting from the amphypathic structure of LA and is manifested in a pronounced change of the adsorbed layer fractal dimension.

The adsorption process at the electrode/electrolyte solution interface goes through similar phases regardless of polarization. Initially, for a very low concentration of solution, the electrode is weakly covered and the adsorbed layer is formed of molecules that are oriented flat on the electrode surface. This layer is not homogeneous, consisting of randomly distributed molecules that are not closely packed together. This situation is reflected in fractal dimension $\mathrm{D} \leq 2$. With an increase in LA concentration corresponding to higher electrode coverage, molecules tend to form irregular patches and then pile up and start to rise, forming an inhomogeneous layer. This is reflected in an increase of fractal dimension to $\mathrm{D}>2.2$. Further concentration increase, reflected in increased coverage that results in stronger molecular interactions, forces the adsorbed molecules to rearrange. In this phase, the fractal dimension from its peak value swiftly decreases as the molecules rearrange from a disordered into a more uniform layer, where the molecules are oriented perpendicularly to the surface with polar heads toward solution. At the end of the process, the adsorbed layer is relatively homogeneous and dense with $\mathrm{D} \approx 2.0$ covering the whole 
electrode surface. Further increase in concentration, up to the investigated $2.5 \times 10^{-4}$ $\mathrm{mol} / \mathrm{dm}^{3}$, does not change this situation, as indicated by the practically constant value of fractal dimension. Hence, we may assume that at the investigated rather high concentration, no bi- or multilayer adsorption of LA occurred. At postmicellar concentration, multilayer formation may be expected, as reported for the sodium oleate adsorption. $^{16}$

The partial electrode coverage calculated from the model of a system with two possibilities of adsorbate orientation and the change of the interaction coefficient with concentration, as calculated by the nonthermodynamic method from apparent isotherms, substantiate these conclusions.

The apparent adsorption isotherms do not show any pronounced changes from which one could firmly deduce that a second order phase change takes place during the adsorption, while the changes in fractal domain are pronounced and very noticeable. Hence, it seems that the method of scaling a mercury electrode, in order to obtain the fractal dimension of the adsorbed layer, provides a sensitive means for the detection of phase changes in adsorption processes studied by ac voltammetry.

Acknowledgement: The authors acknowledge support from the Croatian Ministry of Science and Technology, grants No. 00980303 and No. 00981503. 


\section{- Appendix:}

The adsorption at a mercury electrode is influenced, to a varying degree, by $\pi$-electron interaction with the charges of the electrode surface and molecular interactions. If, as a result of these interactions, the organic molecules can be adsorbed in two different positions, the model of two parallel condensers becomes inapplicable in a general case.

To represent a system in which the organic substance is adsorbed at the electrode in two different positions, the two-condenser model must be replaced by a model consisting of three parallel condensers. Water molecules are between the plates of the first, organic molecules with one orientation between the plates of the second, and molecules in the second orientation between the plates of the third capacitor.

The equation for the electrode charge $\varepsilon$ corresponding to this model is

$$
\varepsilon=\varepsilon_{0}\left(1-\theta_{1}-\theta_{2}\right)+C_{1}\left(\varphi-\varphi_{N 1}\right) \theta_{1}+C_{2}\left(\varphi-\varphi_{N 2}\right) \theta_{2}
$$

where $\theta_{1}$ and $\theta_{2}$ represent the coverage by organic molecules in positions 1 and 2 , respectively; $\mathrm{C}_{1}$ and $\mathrm{C}_{2}$ are the capacities of the double layer with complete surface coverage in positions 1 and $2, \varphi$ is the potential, $\varphi \mathrm{N} 1$ and $\varphi \mathrm{N} 2$ are shifts of p.z.c. in transition from the pure electrolyte solution to $\theta_{1}=1$ or $\theta_{2}=1$, respectively.

Additionally, one must choose two adsorption isotherms connecting $\theta_{1}$ and $\theta_{2}$ with the volume concentration $\mathrm{C}$ of the organic substance, so that the value of $\Gamma$ for an organic substance adsorbed in two positions is written in the forms

$$
\Gamma=\Gamma_{\mathrm{m} 1} \theta_{1}+\Gamma_{\mathrm{m} 2} \theta_{2}
$$

where $\Gamma_{\mathrm{m} 1}$ and $\Gamma_{\mathrm{m} 2}$ are the values of $\Gamma$ when $\theta_{1}=1$ and $\theta_{2}=0$ and $\theta_{1}=0$ and $\theta_{2}=1$, respectively.

The surface coverage is

$$
\theta=\theta_{1}+\theta_{2}
$$


If we assume that the interaction between adsorbed molecules leads to a change of their orientation from flat to perpendicular with an increase of the adsorbate concentration in such a way that the part of the adsorption potential difference due to perpendicularly oriented molecules, i.e. $\theta_{2} \varphi_{\mathrm{N} 2} / \theta$, increases linearly with $\theta$ while the part corresponding to flat oriented molecules $\left(\theta_{1 \varphi N_{1} /} / \theta\right)$ decreases linearly with the surface coverage, then ${ }^{3}$

$$
\frac{\theta \varphi_{N 2}}{\theta}=k_{2} \theta ; \quad \frac{\theta \varphi_{N 1}}{\theta}=k_{1}(1-\theta)
$$

and Eq. (A1) becomes

$$
\varepsilon=\varepsilon_{0}(1-\theta)+C^{1} \theta\left\{\varphi-\left[k_{2} \theta+k_{1}(1-\theta)\right]\right\}
$$

where $\mathrm{k}_{1}$ and $\mathrm{k}_{2}$ are the proportionality constants and it was assumed that $\mathrm{C}_{1}=\mathrm{C}_{2}=\mathrm{C}^{\prime}$.

When $\theta \rightarrow 0$ molecules are adsorbed only in position 1 (flat) and when $\theta \rightarrow 1$ only in position 2 (perpendicular), $\mathrm{k} 1=\varphi_{\mathrm{N}}(0)=\varphi_{\mathrm{N} 1}$ while $\mathrm{k}_{2}=\varphi_{\mathrm{N}}(1)=\varphi_{\mathrm{N} 2}$. Then, from Eq. (A3) it follows that

$$
\theta_{1}=\theta(1-\theta) \quad \text { and } \quad \theta_{2}=\theta^{2}
$$




\section{References}

(1) Frew, N.M. In The sea surface and global change; Liss, P.S., and Duce, R.A., Eds.; University Press: Cambridge, 1997; p. 121.

(2) Romankevich, E.A. Geochemistry of Organic Matter in the Ocean; Springer-Verlag: Berlin, 1984; Chapter 8.

(3) Blank, M.; Miller, I.R. J. Colloid. Interface. Sci. 1968, 26, 26.

(4) Lipkowski, J.; Stolberg, L.; Yang, D.-F.; Pettinger, B.; Mirwald, S.; Henglein, F.; Kolb, D.M. Electrochim. Acta 1994, 39, 1045.

(5) Romanowski, S.; Maksymiuk, K.; Galus, Z. J. Electroanal. Chem. 1995, $385,95$.

(6) Leermakers, F. A.; M.; Nelson, A. J.Electroanal. Chem. 1990, 278, 53.

(7) Nelson, A.; Leermakers, F. A. M. J. Electroanal. Chem. 1990, 278, 73.

(8) De Levie, R., Chem. Rev. 1988, 599, 88,

(9) Becucci, L.; Moncelli, M.R.; Guidelli, R. J. Electroanal. Chem. 1996, 413, 187.

(10) Herrero, R.; Moncelli, M.R.; Becucci, L.; Guidelli, R. J. Electroanal. Chem. 1997, 425,87 .

(11) Bizzotto, D.; Nelson, A. Langmuir 1998, 14, 6269.

(12) Damaskin, B.B.; Petrii, O.A.; Batrakov, V.V. Adsorption of organic compounds on electrodes; Plenum Press: New York, 1971; Chapters 3 and 4.

(13) Jehring, H. Elektrosorptionsanalyse mit der Wechselstrompolarographie Akademie: Verlag, 1974.

(14) Bond, A. M. Modern Polarographic Methods in Analytical Chemistry Marcel Dekker: New York, 1980. 
(15) Ulrich, H-J.; Stumm, W.; Ćosović, B. Environ. Sci. Technol. 1988, 22, 37.

(16) Sotiropoulos, S.; Avranas, A.; Papadopoulos, N. Langmuir 1997, 13, 7230.

(17) Van-Damme H.; Fripiat, J.J. J. Chem. Phys., 1985, 82, 2785.

(18) Ross, S. B.; Smith, D. M.; Hurd, A. J.; Shaefer, D.W. Langmuir, 1988, 4, 977.

(19) Pietronero, L.; Tasatti, E. Fractals in Physics; North Holland: Amsterdam, 1986.

(20) Farin, D.; Avnir, D. Colloids Surf., 1989, 37, 155.

(21) Pines-Rjanski, D.; Huppert, D.; Avnir, D. Chem. Phys. Lett., 1987, 139, 109.

(22) Avnir, D. J. Am.Chem. Soc, 1987, 109, 2931.

(23) Farin, D.; Avnir, D. J. Catal., 1989, 120, 55.

(24) Gutfraind, R.; Shieituch, M. J. Chem. Phys. 1991, 95, 6100.

(25) Avnir, D.; Farin, D. New. J. Chem., 1992, 16, 439.

(26) Pajkossy, T. J. Electroanal. Chem., 1991, 300, 1.

(27) Wandlowski, T.; Pospíšil, L. J. Electroanal. Chem. 1989, 270, 319.

(28) Mandelbrot, B.B. The Fractal Geometry of Nature; Freeman: San Francisco, 1982.

(29) Bockris, J. O’M.; Devanathan, M. A. V.; Müller, K. Proc. R. Soc. London $A, \mathbf{1 9 6 3}, 274,55$.

(30) Rangarajan, S.K. In Specialist Periodical Reports, Electrochemistry, Vol. 7; Thirsk, H.R., Ed.; The Chemical Society: London, 1980; p. 203.

(31) Nikitas, P. J. Electroanal. Chem. 1991, 300, 607.

(32) Schwarzenbach, R.P.; Stierly, R.; Folsom, B.R.; Zeyer, J. Environ. Sci. Technol. 1988, 22, 2632. 
Figure 1. Differential capacity-potential curves for the bulk linoleic acid concentrations: $0.05,0.1,0.2,0.3,1.0$ and $3 \mathrm{mg} / \mathrm{dm}^{-3}$ (curves 2-7). Pure electrolyte (curve 1). Accumulations time 1 min.

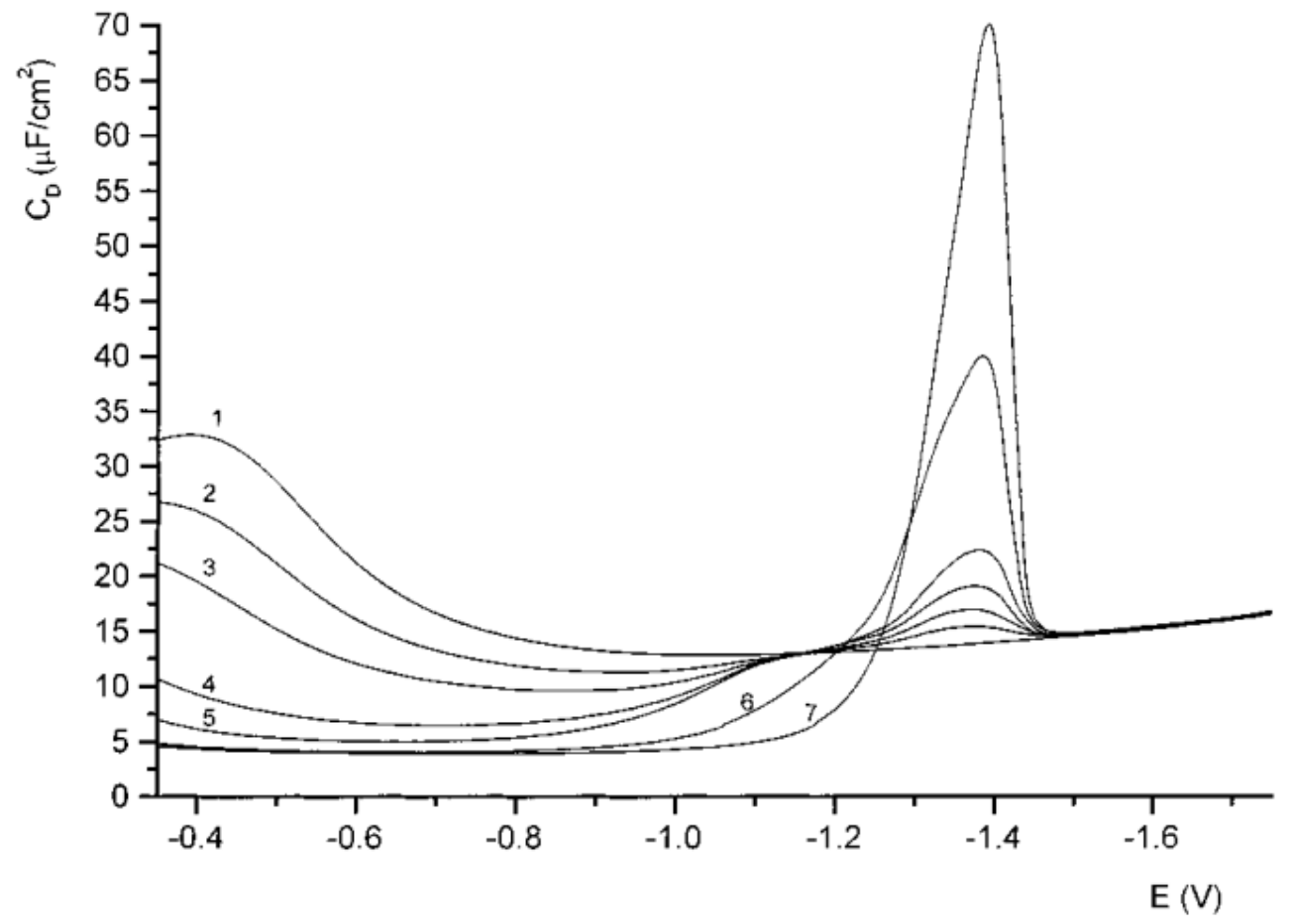

Figure 2. Dependence of differential capacity on bulk linoleic acid concentration for the three potentials, $-0.35,-0.6 .-0.8 \mathrm{~V}$.

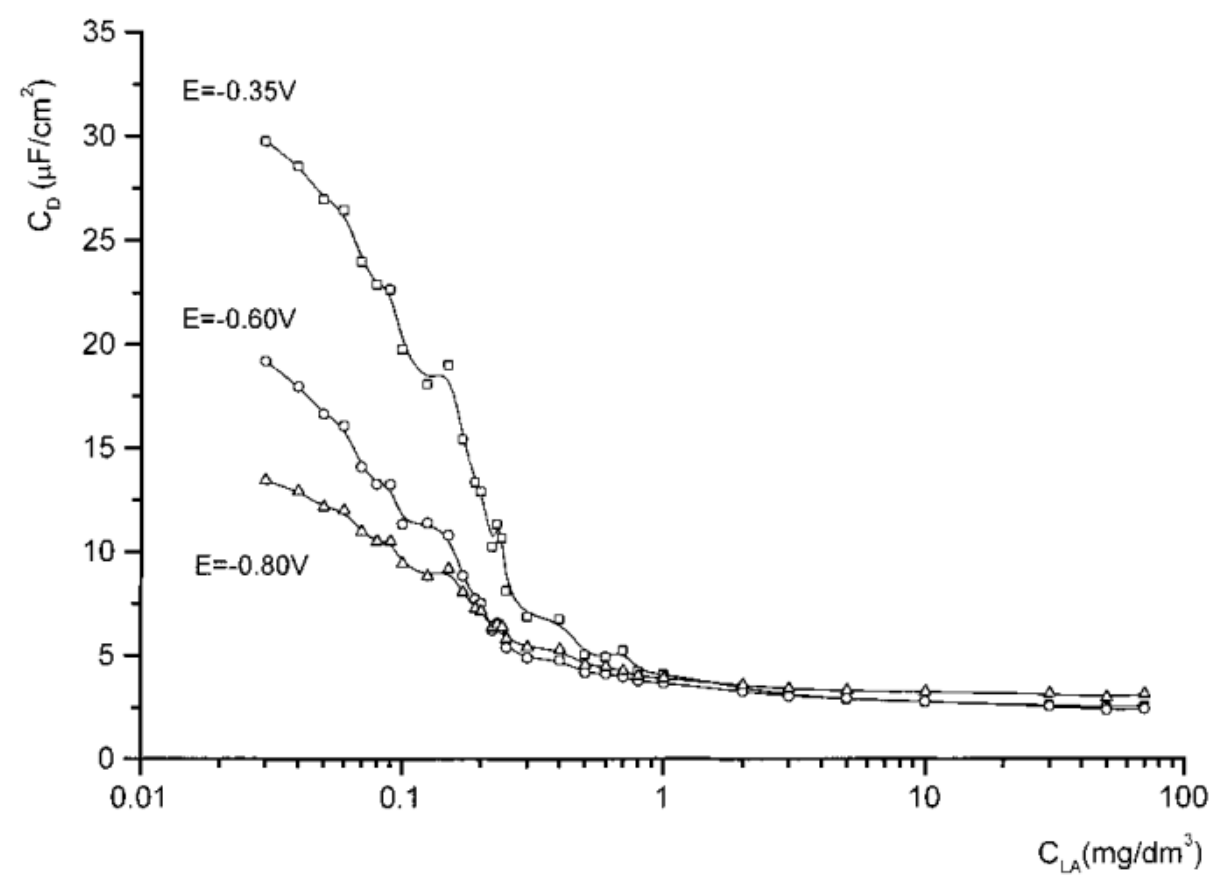


Figure 3. Capacitive current-potential curves, for nine electrode sizes, for electrolyte (curves 1-9) and $0.19 \mathrm{mg} / \mathrm{dm}^{-3}$ linoleic acid (curves $1 *-9 *$ ). Surface areas for $1,1^{*}-9,9 *$ are: $0.88,1.4,1.83,2.22,2.58,2.91,3.23,3.53$ and $3.81 \mathrm{~mm}^{2}$, respectively.

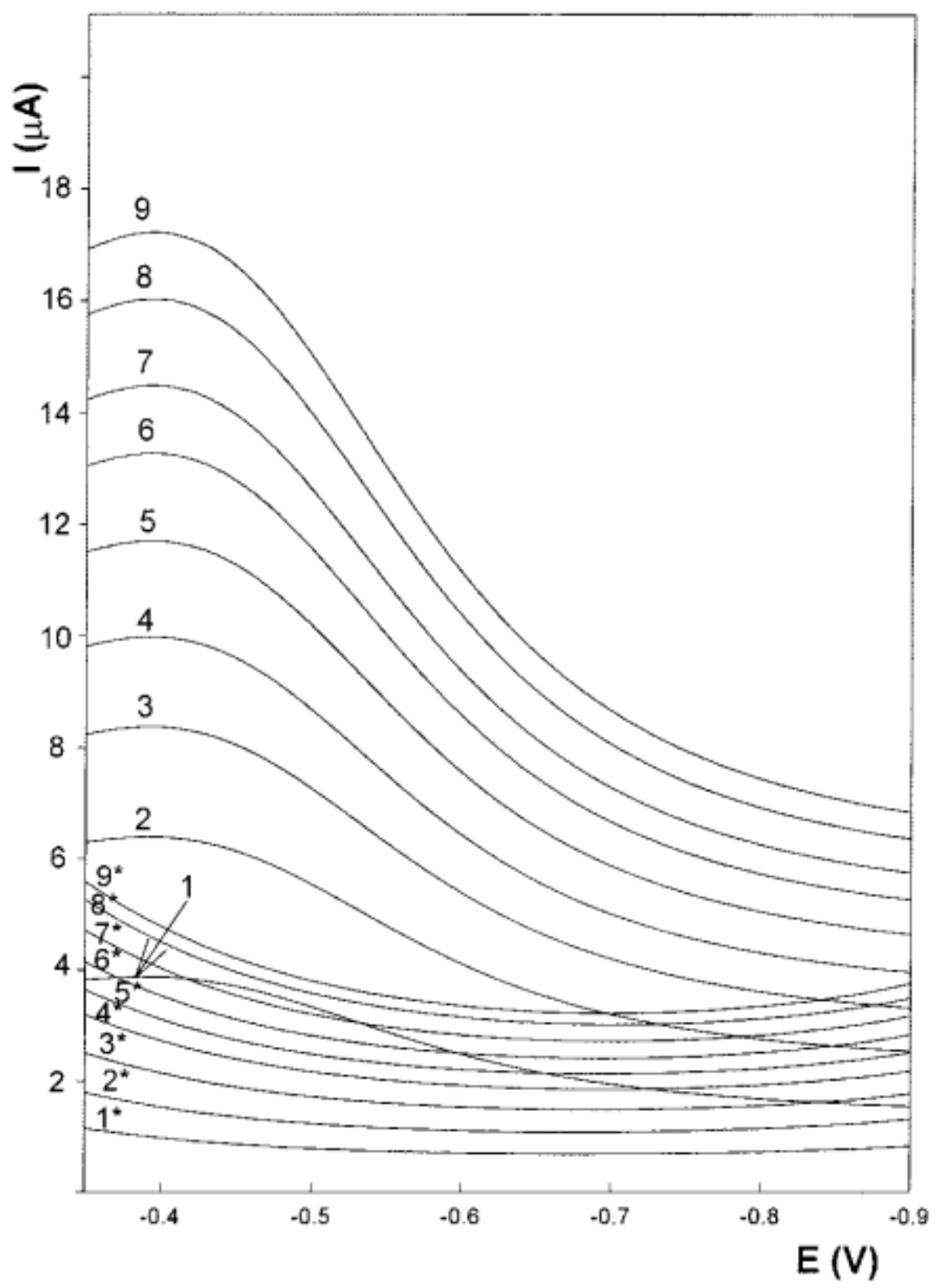


Figure 4. Dependence of the measured capacitive current at $-0.6 \mathrm{~V}$ on the electrode radius (r) for pure electrolyte $(\square)$ and $0.19 \mathrm{mg} / \mathrm{dm}^{3} \mathrm{LA}(\circ)$ with the corresponding linear fit (lines 1 and 2 for pure electrolyte and $0.19 \mathrm{mg} / \mathrm{dm}^{3} \mathrm{LA}$, respectively). D and R are the fractal dimension and the regression coefficient, respectively.

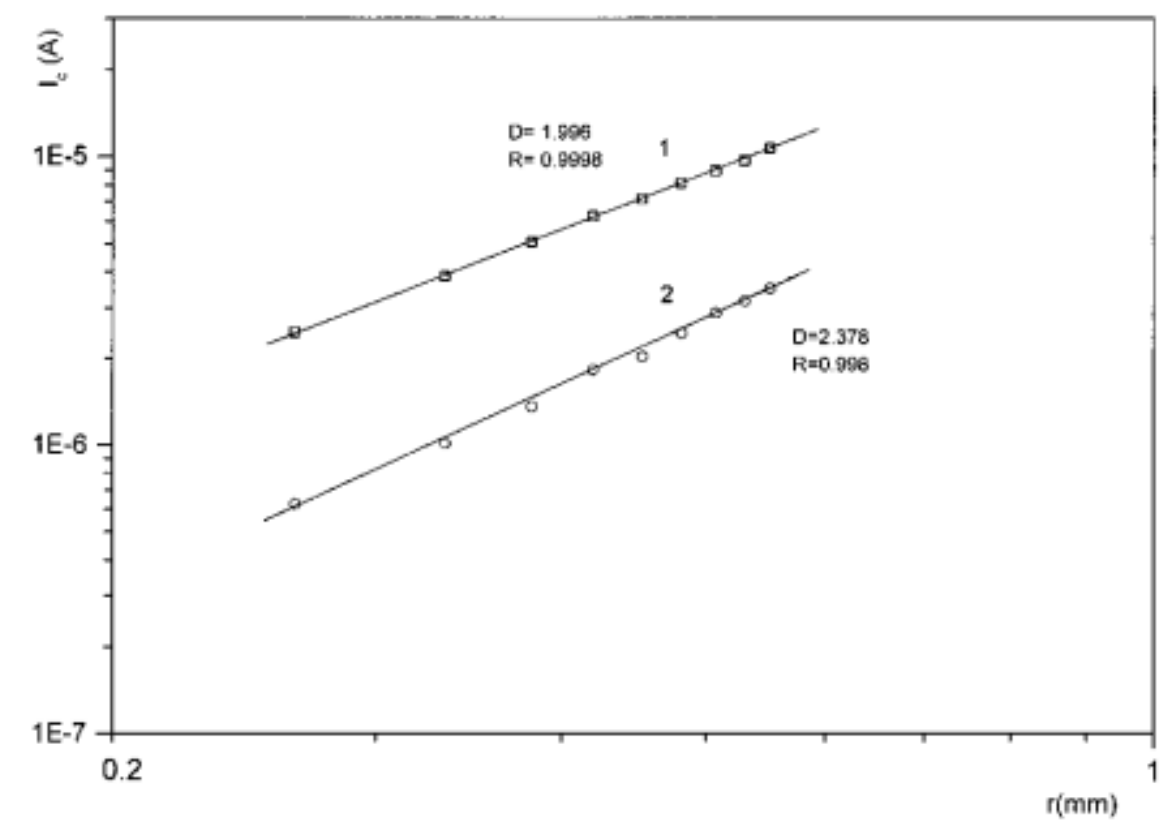


Figure 5. (a) Dependence of the fractal dimension on bulk LA concentration for the electrode potential $\mathrm{E}=-0.6 \mathrm{~V}$; (b) The corresponding total fractional electrode coverage $(\theta)$ and fractional coverage with flat $\left(\theta_{1}\right)$ and perpendicularly oriented $\left(\theta_{2}\right)$ molecules; and (c) Variation in the coefficient of lateral interaction. Symbols denote measurement points while lines represent the fit with B-spline. Details in text.

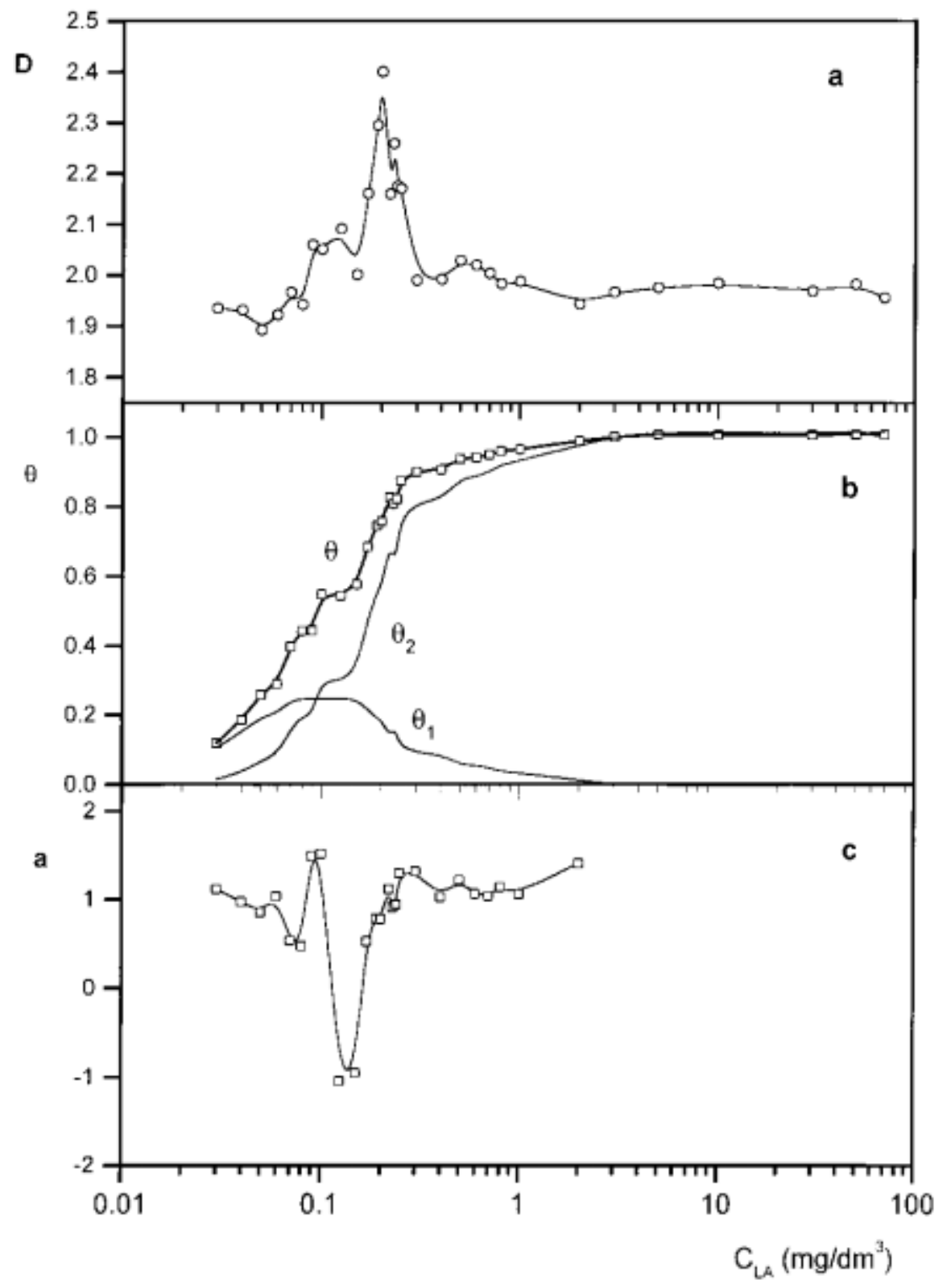


Figure 6. Variation of the fractal dimension (a); fractional electrode coverage (b); and coefficient of lateral interaction (c) with bulk LA concentration for different electrode polarities: $-0.35 \mathrm{~V}$ (curves 1), $-0.60 \mathrm{~V}$ (curves 2) and $-0.80 \mathrm{~V}$ (curves 3). Symbols denote measurement points while lines represent the fit with B-spline.

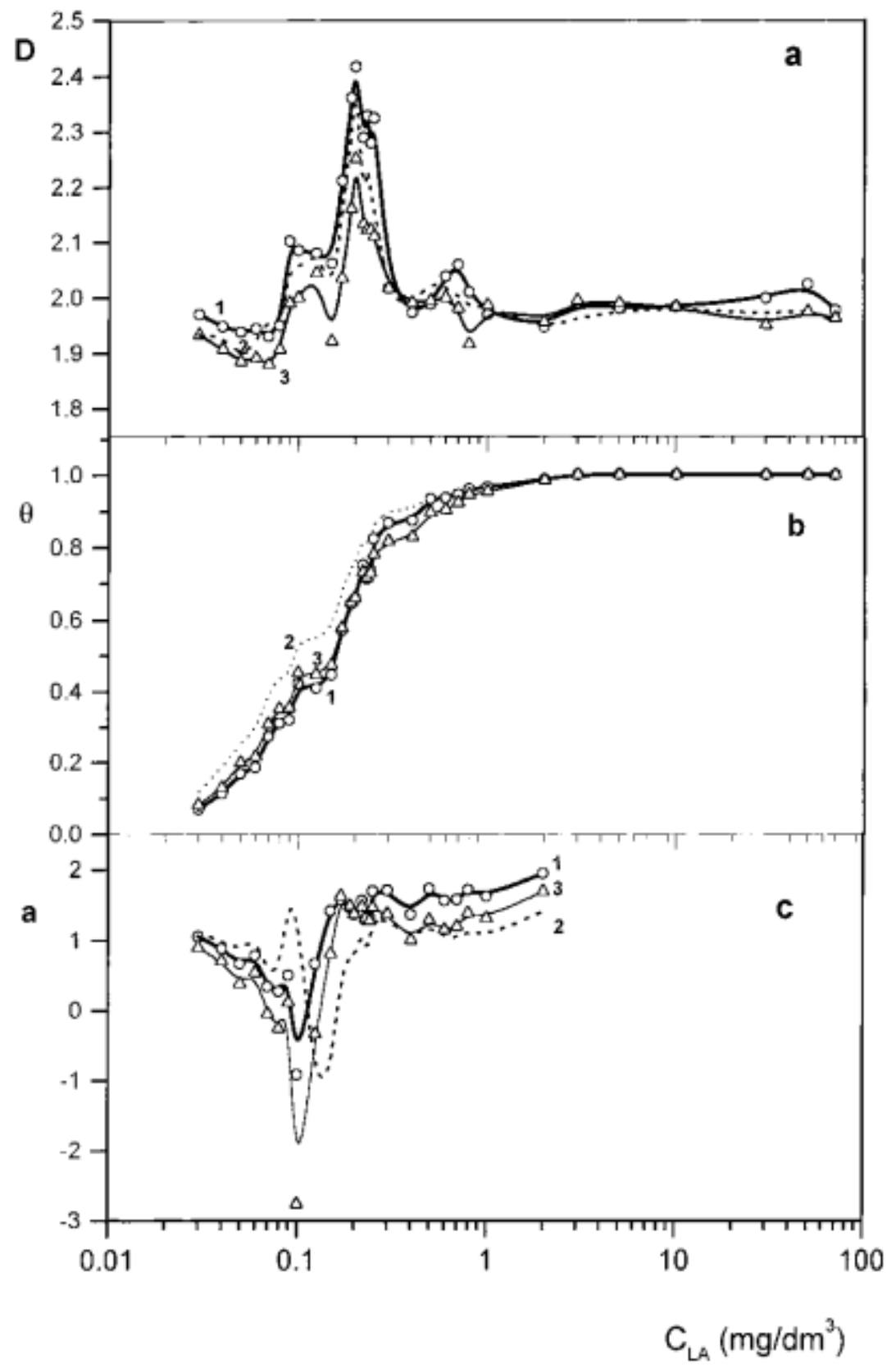


Figure 7. Dependence of the fractional electrode coverage with flat $\left(\theta_{1}\right)$ and perpendicularly oriented $\left(\theta_{2}\right)$ molecules on bulk LA concentration for (a) approx. neutral $(\mathrm{E}=-0.60 \mathrm{~V})$ and positively charged electrodes $(\mathrm{E}=-0.35 \mathrm{~V})$; and (b) for approx. neutral and negatively charged electrodes $(\mathrm{E}=-0.80 \mathrm{~V})$.

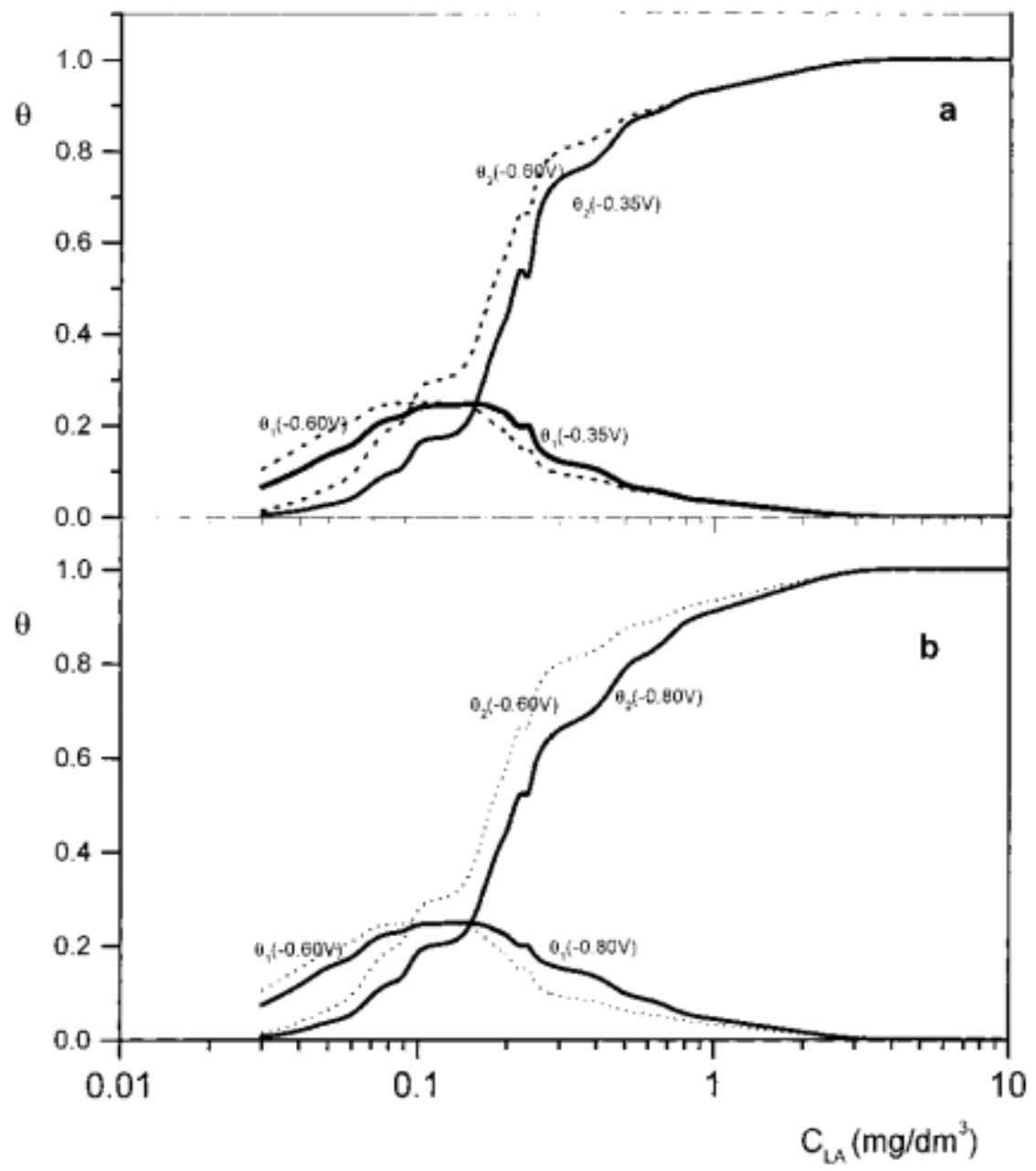

